\title{
THE CERN POLARIZED ATOMIC BEAM TARGET PROJECT
}

\author{
UA6 COLLABORATION*)
}

L. Dick

CERN, Geneva, Switzerland and University of Milan, Italy

W. Kubischta

CERN, Geneva, Switzerland

\begin{abstract}
The UA6 experiment at the CERN p p Collider, currently running with an unpolarized hydrogen cluster target, is considering the development of a polarized atomic hydrogen-beam target. The design goal is a free-jet target with a thickness of up to $1 \times 10^{13}$ atoms $/ \mathrm{cm}^{2}$ and a polarization of $>90 \%$. The compatibility of the target with the Collider operation is of prime importance, since the experiment runs in parasitic mode. We discuss the basic parameters and the atom optical calculations for an atomic beam using superconducting sextupoles.
\end{abstract}

To be published in:

Proc. $8^{\text {th }}$ Int. Symp. on High-Energy Spin Physics

(Workshop on Polarized Gas Targets for Storage Rings)

*) Bologna-CERN-Lausanne-Michigan-Milan-Rockefeller-Trieste-Udine 


\title{
THLE CLEN POLARILED A'TOMIC BLAM IARGIL'T PROJLC"I
}

UA6 Collaboration*)

L. Dick

CERN, Geneva, Switzerland and University of Milan, Italy

W. Kubischta

CERN, Geneva, Switzerland

\begin{abstract}
The UA6 experiment at the CERN p $\vec{p}$ Collider, currently running with an unpolarized hydrogen cluster target, is considering the development of a polarized atomic hydrogenbeam target. The design goal is a free-jet target, with a thickness of up to $1 \times$ $10^{13}$ atoms $/ \mathrm{cm}^{2}$ and a polarization of $>90 \%$. The compatibility of the target with the Collider operation is of prime importance, since the experiment runs in parasitic mode. We discuss the basic parameters and the atom optical calculations for an atomic beam using superconducting sextupoles.
\end{abstract}

\section{INTRODUCTION}

Since 1984 , a cluster-beam target ${ }^{1}$ has been used parasitically during the $p \bar{p}$ Collider periods of the CERN Super Proton Synchrotron (SPS) (Experiment UA6) ${ }^{2}$, and the compatibility of such an experiment with a hadron collider has been clearly demonstrated. It has therefore been proposed to replace this target by a polarized atomic hydrogen-beam target after the end of the present program ${ }^{3}$. (A polarized jet prototype ${ }^{4}$, built in view of an earlier proposal ${ }^{5}$, was finally not installed, since priority was given to the $\mathrm{p} \overline{\mathrm{p}}$ physics with the cluster jet.)

The basic parameters of the beams in the CERN p p Collider are given in table I, as far as they are relevant to the target operation.

Table I Main parameters of the Collider beams

$\begin{array}{ll}\text { Energy: } & \text { usually } 315 \mathrm{GeV} \\ \text { Revolution frequency: } & 43.4 \mathrm{kHz} \\ \text { Proton beam: } & 6 \text { bunches of up to } 1.6 \times 10^{11} \mathrm{p} \text { each } \\ \text { Antiproton beam: } & 6 \text { bunches of up to } 8 \times 10^{10} \overline{\mathrm{p}} \text { each } \\ \text { Approx. beam size at target: } & 4 \mathrm{~mm} \text { (horizontal) } \times 2 \mathrm{~mm} \text { (vertical) }\end{array}$

The average pressure in the machine is a few $10^{-10}$ mbar. During cluster-target operation, the pressure within $3 \mathrm{~m}$ of the target rises to about $10^{-6}$ mbar. Theoretically, the average luminosity is reduced by about $5 \%$ owing to the target plus the pressure bump, but in practice this has never been observed, the day-to-day variation in performance, due to other sources, being more important.

*) Bologna-CERN-Lausanne-Michigan-Milan-Rockefeller-Trieste-Udine 
The experiment now proposed requires targel densitics between $2 \times 10^{12}$ and $1 \times 10^{13}$ atoms $/ \mathrm{cm}^{2}$, assuming a target length of up to 2 or $3 \mathrm{~cm}$. Polarization should be better than $90 \%$, which also puts an upper limit on background pressure (at $10^{-7} \mathrm{mbar}$, the density is $5 \times 10^{9}$ protons $/ \mathrm{cm}^{3}, 10 \mathrm{~cm}$ corresponding to $1 \%$ of the projected polarized target density). Since one part of the experiment would consist of the detection of low-energy recoil particles from elastic scattering, the magnetic field at the target should not be larger than about $100 \mathrm{G}$. We therefore need a beam consisting of state- 1 atoms only. On the other hand, the relatively low field allows also a convenient reversal of polarization by reversing the field direction.

\section{THE TARGET}

As already discussed during the Workshop held in Montana ${ }^{6}$, we intend to use a free, continuous, atomic beam as the target, other possibilities - such as cell or pulsed very cold atoms - not being fully compatible with the environment of the experiment.

The schematic layout of the target is shown in fig. 1. After having been dissociated, the gas passes a nozzle-skimmer combination. Two sextupoles with a 2-4 RF transition in between, select and focus a fraction of state-1 atoms into the target region. After passage through the accelerator beam, the target beam is absorbed in a cryodump.

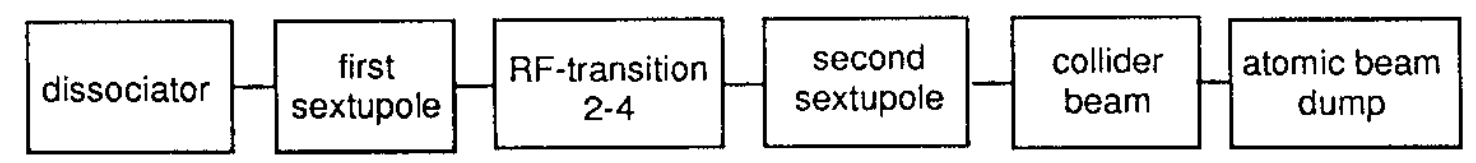

Fig. 1. Schematic layout of atomic-beam target.

With the exception of the RF transition between the magnets, the system closely resembles an optimized, cooled, atomic-beam section for a polarized ion source ${ }^{7}$. Density values of up to $2 \times 10^{12}$ atoms $/ \mathrm{cm}^{3}$ (continuous) within a diameter of about $1 \mathrm{~cm}$ have been reported. Keeping in mind that we work with one state only, we could expect a target thickness (density $\times$ diameter) of about $1 \times 10^{12}$ atoms $/ \mathrm{cm}^{2}$. This is somewhat below the lower limit for our experiment, and we are looking for possibilities to improve it.

\subsection{Atomic beam formation}

We plan to use a nozzle cooled to about $30 \mathrm{~K}$, as published at the Montana Workshop ${ }^{8}$. The reported velocity distributions were very similar to those found at Zurich ${ }^{9}$. For the calculations described in the next paragraph, we have used the distribution function with the most probable velocity, $1100 \mathrm{~m} / \mathrm{s}$, and width at half maximum, $600 \mathrm{~m} / \mathrm{s}$ (Mach number 2.8).

We have also measured the angular distribution of the beam, frecly expanding from the nozzle (no skimmer). The result is shown in fig. 2 together with curves of some powers of cosine. It seems that the data are well represented by a function of the form

with $n=5$.

$$
\mathrm{j}(\theta) \sim \cos ^{11} \theta,
$$




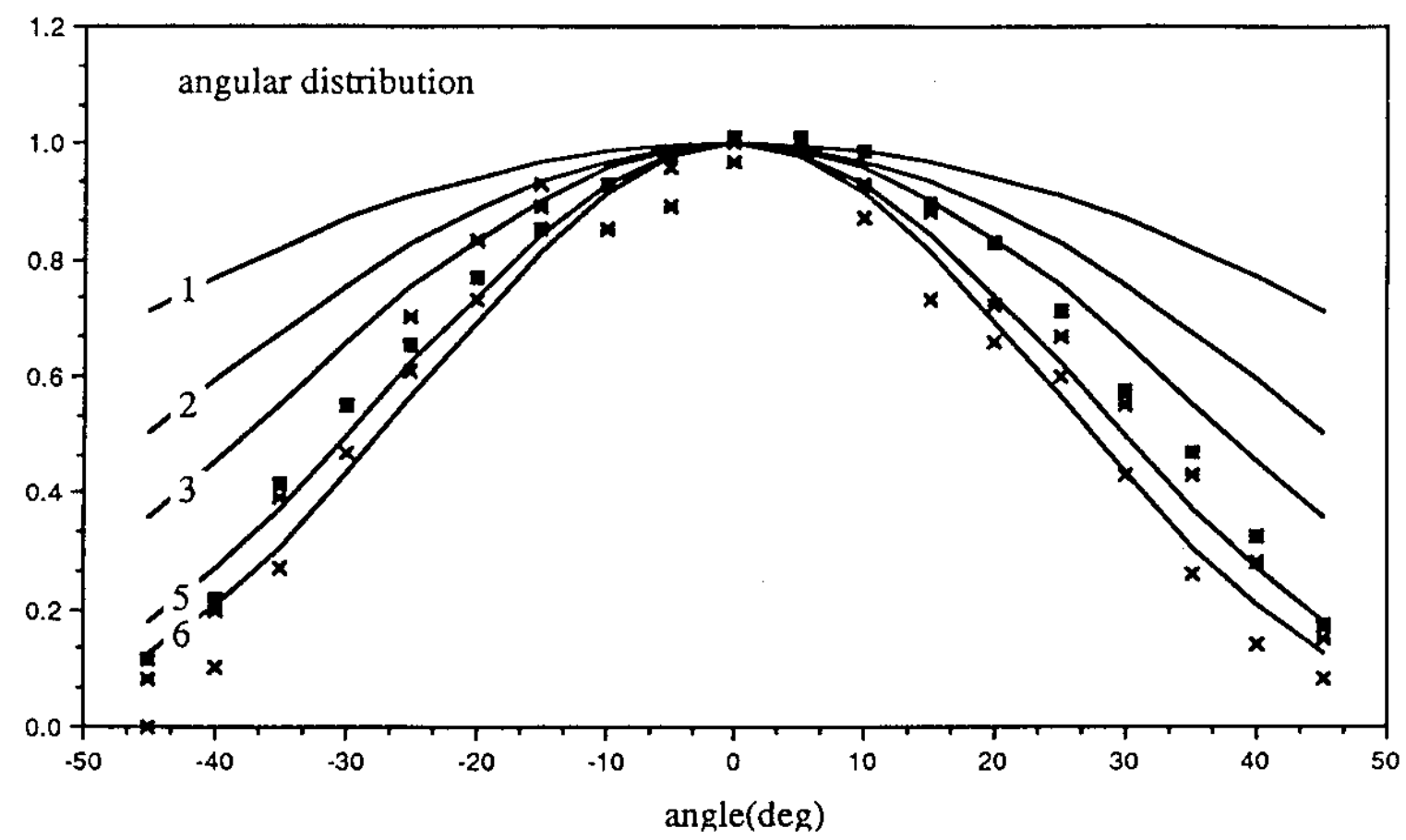

Fig. 2. Angular distribution of nozzle beam (parameter: exponent of $\cos \theta$ ).

\subsection{The magnet system}

The magnet system has three functions:

- to accept a large fraction of the atoms in the beam;

- to transport as many of these as possible to the target point;

- to climinate the unwanted states. by

The maximum angle accepted by an infinitely long constant-aperture sextupole is given

$$
\sin ^{2} \bar{\theta}=\frac{2 \mu \mathrm{B}_{1}}{\mathrm{~m} v^{2}}
$$

with $B_{p}$ the pole-tip field and $v$ the velocity of the atom. The angular acceptance (for a point on the axis) is then

$$
\hat{\mathrm{a}}(\mathrm{v})=\int_{0}^{2 \pi} \int_{0}^{\bar{\theta}} \sin \theta \mathrm{d} \theta \mathrm{d} \phi=2 \pi(1-\cos \hat{\theta}) \approx \frac{\left.2 \pi \mu \mathrm{B}_{1}\right)}{\mathrm{mv}^{2}} ;
$$

which is proportional to $\mathrm{B}_{\mathrm{n}}$ for a given velocity. Since the pole-tip fields of classical sextupoles are limited to about $0.9 \mathrm{~T}$, we propose to use superconducting sextupoles with pole-tip fields of $4 \mathrm{~T}$ and apertures 60 to $80 \mathrm{~mm}^{*}$.

*) T. Tortschanoff and T. Taylor, from CERN-LEP, have determined the technical feasibility of such magnets. 


\subsection{The atom optics program}

In order to optimize the second function of the system - the transport to the target point - the geometry has to be optimized (internal diametcrs and length of the magnets, and the distance between them), taking into account constraints such as the distance between the second magnet and the target point.

We use a combination of ray-tracing and acceptance-diagram ${ }^{10}$ techniques. For a given velocity and a given starting radius, a ray-tracing program determines the minimal and maximal angle $\left(\theta_{1}, \theta_{2}\right)$ transmitted to the target region. Trajectories start at the nozzle or, to be more correct, at the 'freezing surface', the region of transition from continuum to molecular flow.

The optical quality of the system can then be determind from the average angular acceptance as seen from the nozzle with radius $r_{0}$ :

$$
\langle\mathrm{a}(\mathrm{r})\rangle=\frac{1}{\mathrm{r}_{0}^{2} \pi} 2 \pi \int_{0}^{2 \pi} \int_{0}^{\mathrm{r}_{0}} \mathrm{r} \int_{0}^{\theta_{\lambda}(\mathrm{r}, \mathrm{v})} \sin \theta \mathrm{d} \theta \mathrm{dr} \mathrm{d} \phi
$$

with $\theta_{\Lambda}=\left(\theta_{2}-\theta_{1}\right) / 2$

When we include the properties of the nozzle beam (velocity and angular distribution), the intensity $I_{1}$ reaching the target can be calculated from

$$
I_{t}=\left.I_{0} \frac{2 \pi(n+1)}{\mathrm{F}_{0} \bar{v}}\right|_{0} ^{\infty} \int_{0}^{r_{0}} \int_{0}^{\theta_{2}(\mathrm{r}, \mathrm{v})} \mathrm{vf}(\mathrm{v}) \cos ^{\mathrm{n}} \theta \sin \theta \mathrm{d} \theta \mathrm{rdrdv},
$$

with $\mathrm{I}_{0}$, the intensity of the beam leaving the nozzle, $\mathrm{f}(\mathrm{v})$ the velocity distribution of the form

$$
f(v)=x v^{2} \exp \left[-\frac{\left(v-v_{D}\right)^{2}}{v_{\text {th }}^{2}}\right], \quad \int_{0}^{\infty} f(v) d v=1,
$$

and $\bar{v}$ the mean velocity

$$
\vec{v}=\int_{0}^{\infty} v f(v) d v
$$

In the same way, the mean density (atoms $/ \mathrm{cm}^{3}$ ) and the target thickness (atoms $/ \mathrm{cm}^{2}$ ) can be calculated from

$$
\begin{gathered}
\langle\varrho\rangle=\left.\left.1_{0} \frac{2 \pi(\mathrm{n}+1)}{\mathrm{F}_{0} \overline{\mathrm{v}} \mathrm{F}_{1}} \int_{0}^{\infty}\right|_{0} ^{\mathrm{r}_{0}}\right|_{0} ^{\theta_{N}(\mathrm{r}, \mathrm{v})} \mathrm{f}(\mathrm{v}) \cos ^{\mathrm{n}} \theta \sin \theta \mathrm{d} \theta \mathrm{rdr} \mathrm{dv}, \\
\mathrm{TT}=\mathrm{I}_{0} \frac{2 \pi(\mathrm{n}+1)}{\mathrm{F}_{0} \overline{\mathrm{v}}} \int_{0}^{\infty} \int_{0}^{\mathrm{r}_{0}} \prod_{0}^{\theta_{1}(\mathrm{r}, \mathrm{v})} \frac{2 \mathrm{r}_{1}(\mathrm{v})}{\mathrm{F}_{1}(\mathrm{v})} \mathrm{f}(\mathrm{v}) \cos ^{\mathrm{n}} \theta \sin \theta \mathrm{d} \theta \mathrm{rdrdv},
\end{gathered}
$$


where $r_{1}(v)$ is the radius of the accepted trajectory bundle for velocity $v$ at the target, and $F_{I}(v)$ is its surface.

\subsection{Comparison between a classical and a superconducting magnet system}

In order to determine the merits of a superconducting magnet system relative to those of a classical magnet one, we have compared the two systems shown in figs. 3a,b. System 1 has pole-tip fields of $0.9 \mathrm{~T}$, and the first magnet is partially tapered. System 2 has pole-tip fields of $4 \mathrm{~T}$ and cylindrical bores, a superconducting tapered magnet being too difficult to build.

a)

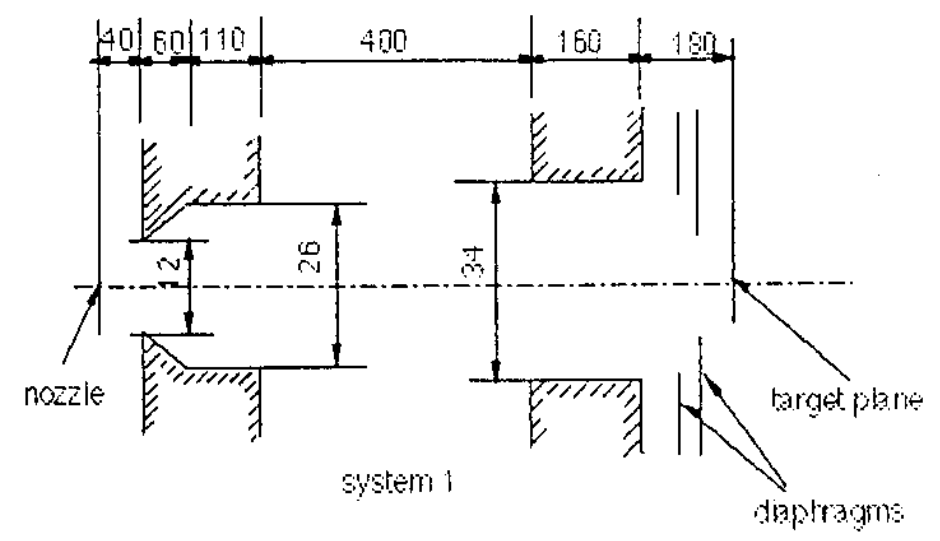

b)

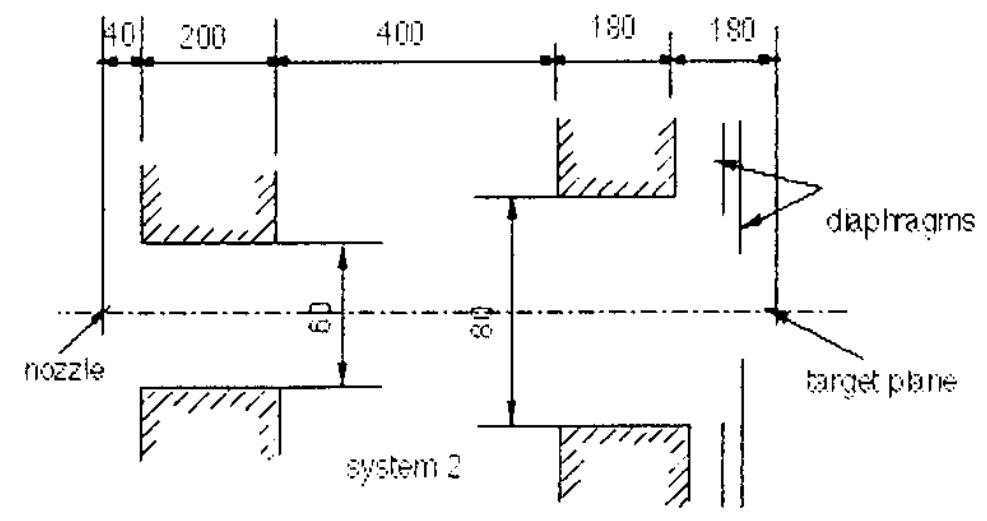

Fig. 3. a) Sextupole system 1 (pole-tip ficld $0.9 \mathrm{~T}$ ); b) sextupole system 2 (pole-tip field $4 \mathrm{~T}$ ).

The most important difference to an ion-source beam is the large angular acceptance of the target region, determined by the two diaphragms before and after the valve separating the atomic beam from the accelerator vacuum system.

Figure 4 shows the ideal acceptance and the actual angular acceptance, as well as the target thickness as a function of atom velocity.

Both the acceptance and the target thickness have maxima at 1100 and about $800 \mathrm{~m} / \mathrm{s}$, about a factor of 4 higher for system 2, the minimum in between being almost identical in 

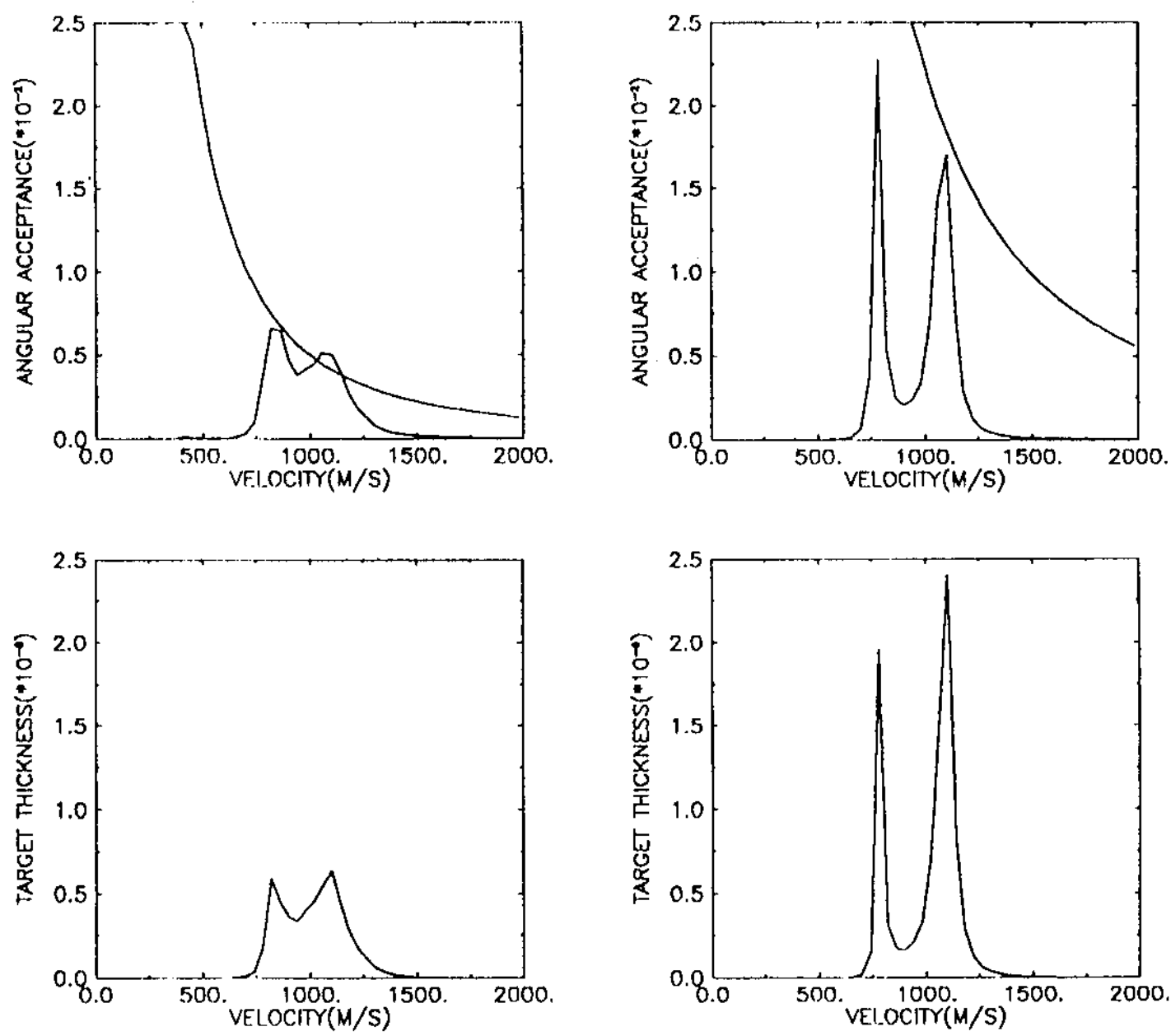

Fig. 4. Angular acceptance (top) and target thickness (bottom) for system 1 (left) and system 2 (right).

both cases. The reason for this behaviour can be understood from the trajectory drawings in fig. 5 .

The magnet lengths chosen are such that for $v=1100 \mathrm{~m} / \mathrm{s}$ the image of the nozzle is at the target plane. For $\mathrm{v} \approx 800 \mathrm{~m} / \mathrm{s}$, a second-order image is formed at the target. For these two velocities, the acceptance is determined essentially by the pole-tip field (therefore the factor of 4), and to a lesser degree by system geometry (in particular, magnet diameters). Between these velocities, the acceptance is mainly a function of system geometry (in particular the distances nozzle to first magnet, and second magnet to target plane). As these values are identical for the two cases, it is not surprising that the acceptance values, too, are almost the same in this velocity range.

The acceptance peaks of system 1 go beyond the 'ideal' curve, a consequence of the tapering of the first magnet.

Target thickness (TT) and polarization values (P) for a few variations of systems 1 and 2 are listed in table II. Version (a) has a nozzle diameter $d_{n}=4 \mathrm{~mm}$ and a last diaphragm diameter $d_{d}=10 \mathrm{~mm}(2 \mathrm{~cm}$ from the target plane). In version (b), the diaphragm is enlarged to $25 \mathrm{~mm}$, and in version (c) also the nozzle diameter is larger $(10 \mathrm{~mm})$.

To calculate the target thickness values for (a) and (b), we have assumed a total gas input of $30 \mathrm{~cm}^{3} / \mathrm{min}=0.5 \mathrm{mbar} \cdot \ell / \mathrm{s}$ (this seems to be the optimal throughput for $30 \mathrm{~K}$ 

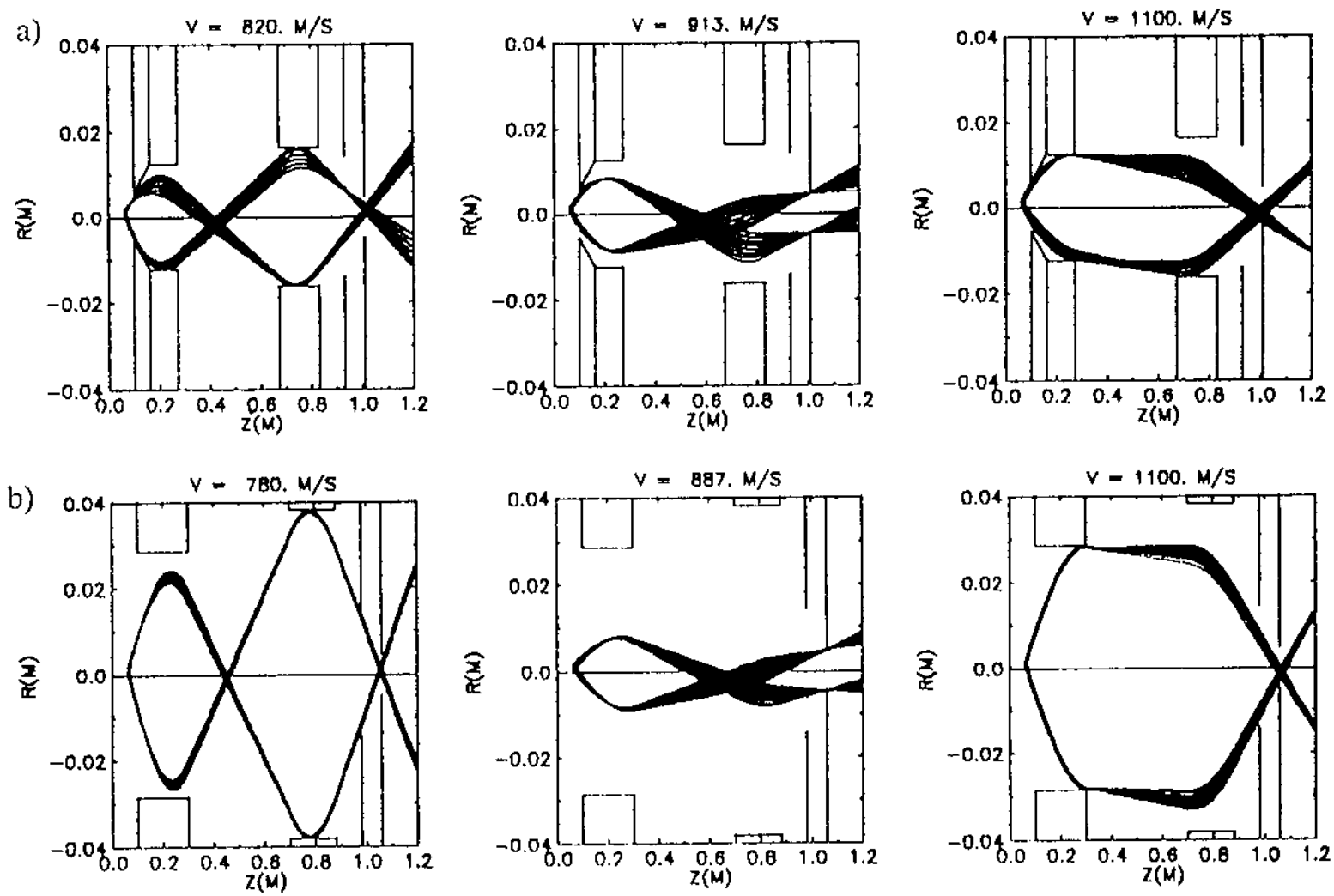

Fig. 5. Trajectories for selected velocities: a) system 1 and b) system 2 .

nozzles). With a dissociation degree of 0.8 , this gives $2 \times 10^{19}$ atoms $/ \mathrm{s}$. As case (c) has a nozzle surface 6.25 times larger than the other two, we assume that from a multiple nozzle, fed from one or several dissociators, it would be possible to produce a gas flow about 4 times larger than that from a standard nozzle.

Taking into account that most of the target thickness is due to the two images, and that the target should anyway not be larger than the Collider beam, we may even use a slit (say, $10 \times 2 \mathrm{~mm}$ ) with a smaller total gas input. The emission properties (velocity and angular distributions) of such an unusual nozzle may be quite different from those of standard nozzles.

Table II Target thickness (atoms $/ \mathrm{cm}^{2}$ ) and polarization for variations of systems 1 and 2 for a given input (atoms/s)

\begin{tabular}{|c|c|c|c|c|c|}
\hline System & $\begin{array}{c}\mathrm{d}_{\mathrm{tl}} \\
(\mathrm{mm})\end{array}$ & $\begin{array}{c}\mathrm{d}_{\mathrm{d}} \\
(\mathrm{mm})\end{array}$ & $\begin{array}{c}\text { Input } \\
\left(\times 10^{19}\right.\end{array}$ & $\begin{array}{c}\mathrm{TT} \\
\left(\times 10^{12}\right)\end{array}$ & $\mathrm{P}$ \\
\hline $\mathrm{la}$ & 4 & 10 & 2 & 1 & 0.97 \\
$\mathrm{lb}$ & 4 & 25 & 2 & 1 & 0.94 \\
$\mathrm{lc}$ & 10 & 25 & 8 & 1.5 & 0.94 \\
$2 \mathrm{a}$ & 4 & 10 & 2 & 2 & 0.94 \\
$2 \mathrm{~b}$ & 4 & 25 & 2 & 2.4 & 0.91 \\
$2 \mathrm{c}$ & 10 & 25 & 8 & 5.7 & 0.93 \\
\hline
\end{tabular}


System 1a gives a TT of $1 \times 10^{12}$ atoms $/ \mathrm{cm}^{2}$, consistent with the ion-source values, taking into account that we have only one state left instead of two. System 2 a gives $2 \times$ $10^{12}$ atoms $/ \mathrm{cm}^{2}$, just enough to compensate for the loss of one state. Enlarging the target diameter or the nozzle and target diameter does not improve system 1, but system 2c produces a TT of $5.7 \times 10^{12}$ atoms $/ \mathrm{cm}^{2}$ with a target length of $25 \mathrm{~mm}$. The total atomic beam flux would be almost $10^{18}$ atoms/s.

Neither system is particularly optimized, since several technological problems remain to be solved. However, the calculations show that the additional complication of having superconducting magnets is justified.

\subsection{The RF transition}

The atomic beam has a diameter of about $6 \mathrm{~cm}$ between the magnets, and we need a radio-frequency transition (2-4) that accepts this beam. We plan to use a rectangular TE102 cavity, which should have a sufficient field homogencity over the required surface.

\subsection{The vacuum system}

The vacuum system is shown schematically in fig. 6. The first stage will have turbomolecular pumps; the other beam stages will be pumped by cryopump panels located

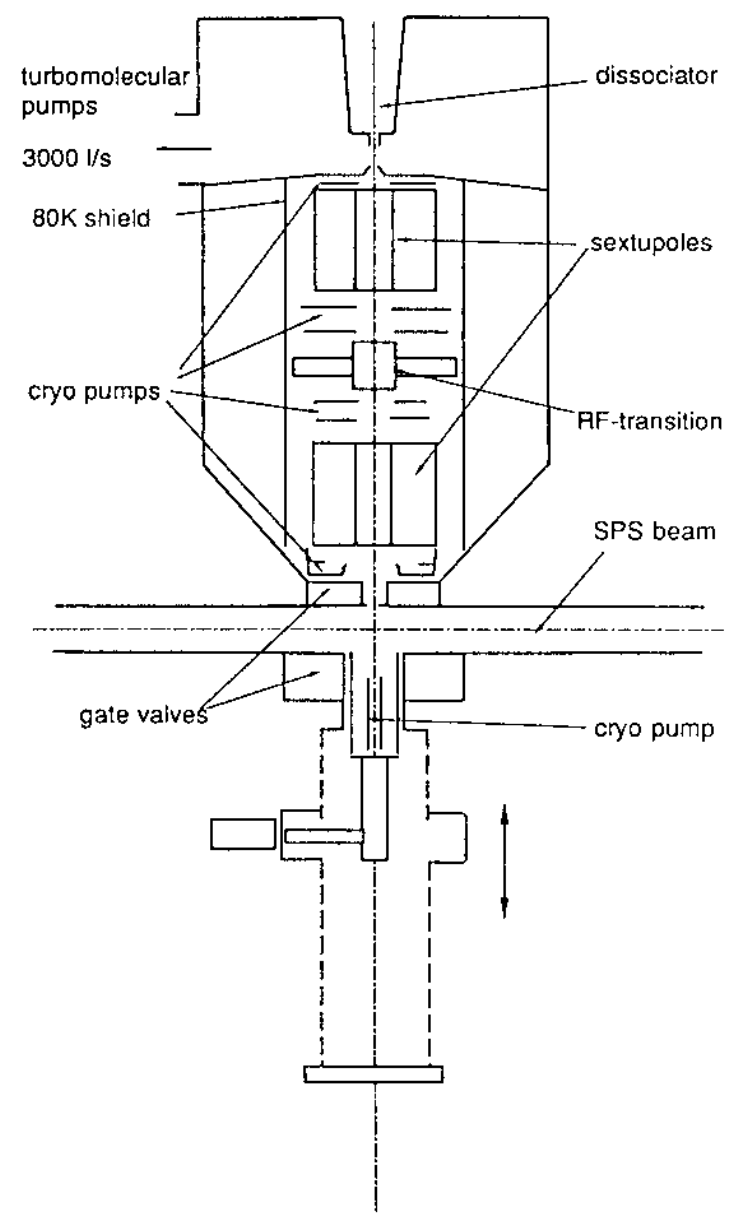

Fig. 6. Vacuum system. 
at the ends and between the magnets. As with the cluster target, the atomic beam production and dump can be separated from the SPS vacuum by all-metal valves, but the lower valve will be of a larger diameter $(150 \mathrm{~mm})$ in order to allow the cryopump to be brought as close as possible to the target region. This assures the highest pumping speed for all gas (in the beam or diffuse) coming from the last diaphragm.

\section{CONCLUSIONS}

With the present cooled, continuous, atomic beams and the 'classical' magnets (pole-tip field $<1 \mathrm{~T}$ ), target densities of not much more than $10^{12}$ atoms $/ \mathrm{cm}^{2}$ can be achieved under our conditions. With superconducting sextupoles, a target thickness up to a factor of 5 to 10 larger can be expected. This assumes unusual nozzles: either a large nozzle with an input four or five times more than that of the standard nozzle, or a slit nozzle, producing an image adapted to the accelerator beam. The target beam would have a dimension of 2 to $2.5 \mathrm{~cm}$ in the direction of the Collider beam.

\section{Acknowledgements}

We would like to thank T. Taylor and T. Tortschanoff for their advice on the technical feasibility of the superconducting sextupoles. The angular distribution measurements have been made by J.C. Berney.

\section{REFERENCES}

1. A. Bernasconi et al. (UA6 Collab.), Phys. Lett. 20613, 163 (1988).

2. L. Dick and W. Kubischta (UA6 Collab.), Physics with jet targets at the SPS p $\bar{p}$ Collider, in Hadronic physics at intermediate energy, T. Bressani and R.A. Rici, eds. (Elsevier Science Publishers, Amsterdam, 1986).

3. Bologna-CERN-Lausanne-Michigan-Milan-Rockefeller-Trieste-Udine Collab., Study of spin effects in $\bar{p}$ and $p p$ interactions at the SPS using a polarized atomic hydrogen beam target, Letter of Intent CERN/SPSC/88-9, SPSC/1168, 16 April 1988.

4. L. Dick, J.B. Jeanneret, W. Kubischta and J. Antille, Proc. Int. Symp. on High-Energy Physics with Polarized Beams and Polarized Targets, Lausanne, 1980 (Experimentia Supplementum 39, Birkhäuser Verl., Basle-Boston-Stuttgart, 1981), p. 212.

5. J. Antille et al. (CERN-Lund-Rockefeller Collab.), Study of spin effects in pp reactions at the SPS using a polarized atomic hydrogen-jet target, Proposal CERN/SPSC/77-71, SPSC/P88, 26 August 1977.

6. L. Dick and W. Kubischta, Proc. Int. Workshop on Polarized Sources and Targets, Montana, 1986 [Helv. Phys. Acta 59 (1986)], p. 584.

7. P.A. Schmelzbach, D. Singy, W. Grüebler and W.Z. Zhang, Nucl. Instrum. Methods A251, 407 (1986).

8. J.C. Berney, L. Dick and W. Kubischta, same Proceedings as Ref. 6, p. 578.

9. P.A. Schmelzbach et al., Proc. 6th Int. Symp. on Polarization Phenomena in Nuclear Physics, Osaka, 1985 [Suppl. J. Phys. Soc. Japan 55, 1060 (1986)].

10. W.Z. Zhang, P.A. Schmelzbach, D. Singy and W. Grüebler, Nucl. Instrum. Methods A240,229(1985). 